\title{
High-energy factorization in QCD and minimal subtraction scheme is
}

\author{
S. Catani ${ }^{a, 1}$, M. Ciafaloni ${ }^{b, c}$ and F. Hautmann ${ }^{\text {b,d }}$ \\ a Theory Division, CERN, CH-1211 Geneva 23, Switzerland \\ b Dipartimento di Fisica, Università di Firenze, I-50125 Florence, Italy \\ c INFN, Seztone di Firenze, I-50125 Florence, Italy \\ d DESY, W-2000 Hamburg 52, Germany
}

Recerved 16 March 1993

Edıtor: R. Gatto

\begin{abstract}
The relationship between high-energy factorization and the factorization theorem of mass singularities is fully established by performing a complete resummed calculation in dimensional regularization. This result is achieved by deriving and solving a new evolution equation for the gluon-gluon forward scatterıng amplitude at high energy in $n$ space-tıme dimensions. This equation reduces to the Balıtskii-Fadin-Kuraev-Lipatov equation in the limiting case of four dimensions.
\end{abstract}

\section{Introduction}

The present and next generations of hadron colliders involve an increasing centre-of-mass energy $\sqrt{S}$. Hadron interactions in this kinematical regime are of the utmost interest both for precise quantitative tests of QCD and for studying new physics within the standard model (top and Higgs production) and beyond. The problem thus arises of providing reliable theoretical predictions for these processes. In particular, one has to address the issue of how to compute, in perturbative QCD, hard-scattering cross sections (i.e. cross sections dominated by large values of transferred momentum $\left\langle p_{\perp}^{2}\right\rangle=Q^{2} \gg \Lambda^{2}, \Lambda$ being the QCD scale) at very high energy $\left(S \gg Q^{2}\right)$. For these processes involving two very different large scales $\left(S \gg Q^{2} \gg \Lambda^{2}\right)$ or equivalently a small value of the ratio $x=Q^{2} / s$, the standard renormalization group approach to factorization [1] has to be reconsidered on the basis of theoretical and phenomenological motivations. On the theoretical side, renormaliza-

\footnotetext{
* Research supported in part by the Italıan Ministero della Unıversità e Ricerca Scıentıfica.

1 On leave of absence from INFN, Sezıone dı Firenze, Florence, Italy.
}

tion group factorization applies to hard scattering in the Bjorken limit ( $x=Q^{2} / S$ fixed, $Q^{2} \rightarrow \infty$ ) whilst the hard scattering is now approaching the Regge regime ( $Q^{2}$ fixed, $x=Q^{2} / S \rightarrow 0$ ). On the phenomenological side, renormalization group factorization of mass singularities leads to large logarithmic corrections $\left(\alpha_{\mathrm{s}} \ln x\right)^{n}$ in each $n$th perturbative order, which may spoil the convergence of the perturbative expansion in the QCD coupling $\alpha_{\mathrm{s}}$.

The QCD factorization for small- $x$ processes has been recently investigated and a new approach, which we called $k_{\perp}$ - or high-energy-factorization theorem [2], has been set up. The high-energy factorization theorem provides a smooth interpolation between renormalization group factorization of mass singularities and Regge factorization; it allows one to resum the leading small- $x$ contributions $\left(\alpha_{\mathrm{s}} \ln x\right)^{n}$ to all orders in perturbation theory both for the structure functions and the hard cross section factor (coefficient function).

In this paper we emphasize the consistence of highenergy factorization and mass singularity factorization and we compute the appropriate coefficient function in the minimal subtraction scheme to all orders in the effective expansion parameter $\alpha_{\mathrm{s}} \ln x$. The general results of the high-energy factorization theo- 
rem are reviewed in section 2 where, as an example, we discuss the case of heavy-flavour photoproduction. The precise relation between the two kinds of factorization is addressed in section 3 by performing calculations in dimensional regularization and presenting the corresponding master equation for the gluon-gluon forward scattering amplitude. Finally, section 4 is devoted to establishing the correspondence between the high-energy factorization and the standard procedure of mass singularity factorization in the modified minimal subtraction scheme.

\section{High energy factorization and resummation}

To be definite let us consider the simplest small- $x$ process initiated by hard gluon interactions at the Born level, namely the heavy flavour photoproduction process (fig. 1a)

$\gamma\left(p_{1}\right)+h(p) \rightarrow Q\left(p_{3}\right)+\bar{Q}\left(p_{4}\right)+X$.

In this case the kinematic scale $x$ has to be identified with the ratio $\rho=4 M^{2} / S$, where $M \gg A$ is the heavyflavour mass.

According to the high-energy factorization theorem, the photon-hadron cross section $\sigma$ is given by (fig. lb) [2]

$$
\begin{aligned}
& 4 M^{2} \sigma\left(\rho, M^{2}\right)=\int \mathrm{d}^{2} \boldsymbol{k} \\
& \times \int_{0}^{1} \frac{\mathrm{d} z}{z} \hat{\sigma}\left(\rho / z, \boldsymbol{k}^{2} / M^{2} ; \alpha_{\mathrm{s}}\left(M^{2}\right)\right) \mathscr{F}(z, \boldsymbol{k}) .
\end{aligned}
$$

Here $\hat{\sigma}$ is the basic high-energy hard cross section for the subprocess $\gamma+g(k) \rightarrow Q \bar{Q}$, computed by coupling the incoming off-shell (essentially transverse $k^{2} \simeq-k^{2}$ ) gluon to high-energy partons via eikonal vertices. In spite of the gluon off-shellness, $\hat{\sigma}$ is gaugeinvariant and computable, as a function of the gluon transverse momentum $\boldsymbol{k}$, in fixed-order perturbation theory. Explicit expressions for all the relevant heavyflavour production processes, namely photoproduction [2], hadroproduction [3-5] and leptoproduction $[3,6]$, are available in the literature.

On the other hand, $\mathscr{F}(x, \boldsymbol{k})$ is the unintegrated

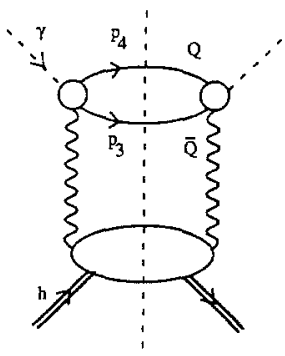

(a)

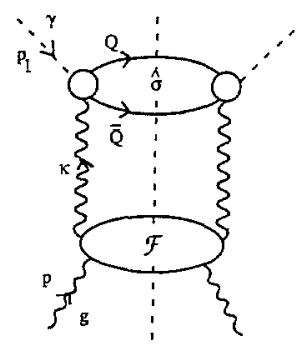

(b)
Fig. 1. (a) The heavy-flavour photoproduction process and (b) its factorized structure at high energy.

gluon $\# 1$ structure function of the hadron, defined as the probability for a gluon to have longitudinal momentum fraction $x$ associated with a total emitted transverse momentum $k$. The full gluon structure function $G\left(x, \mu^{2}\right)$ is obtained by $k_{\perp}$-integration:

$G\left(x, \mu^{2}\right) \equiv \int_{0}^{\mu^{2}} \mathbf{d}^{2} \boldsymbol{k} \mathscr{F}(x, k)$.

Thus $\mathscr{F}(x, \boldsymbol{k})$, as much as $G\left(x, \mu^{2}\right)$, is a universal (i.e. independent of the hard process) parton density of the hadron, which, although not fully computable in perturbation theory, can be experimentally measured in a single process and used via the $k_{\perp}$-factorization formula (2) to make predictions for any other process.

The high-energy factorization in eq. (2) is $k_{\perp}$-dependent and reduces to the leading-order collinear factorization for $S \gg M^{2} \gg k_{\perp}^{2}$. However it holds also for $S \gg k_{\perp}^{2} \sim M^{2}$, i.e. for any value of $k_{\perp} / M$. Since the hard cross section $\hat{\sigma}\left(\rho, \boldsymbol{k}^{2} / M^{2} ; \alpha_{\mathrm{s}}\right)$ is well behaved at high energy (i.e. no enhancement logarithmic factor $\ln \rho$ is present in $\hat{\sigma}$ ), the large perturbative contributions $\left(\alpha_{s} \ln \rho\right)^{n}$ in the cross section (2) are generated precisely by the $k_{\perp}$-integration from the ones in the gluon density $\mathscr{F}(z, \boldsymbol{k})$. Moreover, $\mathscr{F}(x$, $k$ ) being related to gluon structure function $G$ in eq. ( 3 ), its $\boldsymbol{k}$-dependence is computable in perturbation theory via renormalization group equations. Therefore one can explicitly perform the $k_{\perp}$-integration in

\#1 We are considering the case of a single parton species, namely gluons, which dominates the heavy-flavour production process. The generalization to other parton channels is straightforward $[3,7]$ 
(2), which yields automatically the resummation of the leading high-energy logarithms $\left(\alpha_{\mathrm{s}} \ln \rho\right)^{n}$.

To be more precise, let us introduce the $N$-moments of the cross section (2) for fixed $M$

$\sigma_{N}\left(M^{2}\right) \equiv \int_{0}^{1} \mathrm{~d} \rho \rho^{N-1} \sigma\left(\rho, M^{2}\right)$,

and the analogous moments for $\mathscr{F}$. From the customary renormalization group factorization we have $\$ 2$

$\mathscr{F}_{N}(\boldsymbol{k})=\frac{\gamma_{N}\left(\alpha_{\mathrm{s}}\right)}{\pi \boldsymbol{k}^{2}}\left(\frac{\boldsymbol{k}^{2}}{\mu^{2}}\right)^{\gamma_{N}\left(\alpha_{\mathrm{s}}\right)} G_{N}\left(\mu^{2}\right)$,

where $\mu^{2}$ is an arbitrary factorization scale, which has to be chosen on the order of the hard scale $M^{2}$ for applications to heavy-flavour production.

The gluon anomalous dimension $\gamma_{N}\left(\alpha_{\mathrm{s}}\right)$ is computable in QCD perturbation theory and has a wellknown behaviour for $N \rightarrow 0$ (the small- $x$ limit corresponds to $N \rightarrow 0$ in moment space, 1.e. $\ln x$ terms lead to poles in $1 / N$ ), which can be traced back from the work of Balitskii, Fadin, Kuraev and Lipatov [8-10]. More precisely the BFKL anomalous dimension is obtained by the following implicit equation $\left(\bar{\alpha}_{\mathrm{s}} \equiv\right.$ $\left.C_{\mathrm{A}} \alpha_{\mathrm{s}} / \pi\right)$ :

$1=\frac{\bar{\alpha}_{\mathrm{s}}}{N} f\left(\gamma_{N}\left(\alpha_{\mathrm{s}}\right)\right)$,

where $f(\gamma)$ is expressed in terms of the Euler $\psi$ function

$f(\gamma)=2 \psi(1)-\psi(\gamma)-\psi(1-\gamma)$.

By solving eq. (6) in power series of the coupling constant one resums the leading $\left(\alpha_{\mathrm{s}} / N\right)^{k}$ contributions for the gluon anomalous dimension to all orders in $\alpha_{\mathrm{s}}$. The first perturbative terms are $(\zeta(n)$ is the Riemann zeta-function)

$$
\begin{aligned}
& \gamma_{N}\left(\alpha_{\mathrm{s}}\right)=\frac{\bar{\alpha}_{\mathrm{s}}}{N}+2 \zeta(3)\left(\frac{\bar{\alpha}_{\mathrm{s}}}{N}\right)^{4} \\
& +2 \zeta(5)\left(\frac{\bar{\alpha}_{\mathrm{s}}}{N}\right)^{6}+\mathrm{O}\left(\left(\frac{\bar{\alpha}_{\mathrm{s}}}{N}\right)^{7}\right) .
\end{aligned}
$$

\#2 In the following we limit ourselves to the case of a fixed coupling constant $\alpha_{\mathrm{s}}$. In fact, the running coupling effects lead to subleading $\alpha_{\mathrm{s}}\left(\alpha_{\mathrm{s}} \ln x\right)^{n}$ contributions and can thus be neglected in our leading-logarıthmic analysis. We shall comment on the introduction of the running coupling at the end of the paper.
Taking the $N$-moments of the $k_{\perp}$-factorization formula (2) and using the renormalization group equation (5) one obtains

$$
\begin{aligned}
& 4 M^{2} \sigma_{N}\left(M^{2}\right)=C_{N}\left(\alpha_{\mathrm{s}}, M^{2} / \mu^{2}\right) G_{N}\left(\mu^{2}\right), \\
& C_{N}\left(\alpha_{\mathrm{s}}, M^{2} / \mu^{2}\right)=h_{N}\left(\gamma_{N}\left(\alpha_{\mathrm{s}}\right)\right)\left(\frac{M^{2}}{\mu^{2}}\right)^{\gamma_{N}\left(\alpha_{\mathrm{s}}\right)},
\end{aligned}
$$

where the function $h_{N}(\gamma)$ is given by the following $k_{\perp}$-transform of the hard cross section $\hat{\sigma}$ :

$h_{N}(\gamma) \equiv \gamma \int_{0}^{\infty} \frac{\mathrm{d} \boldsymbol{k}^{2}}{\boldsymbol{k}^{2}}\left(\frac{\boldsymbol{k}^{2}}{M^{2}}\right)^{\gamma} \hat{\sigma}_{N}\left(\boldsymbol{k}^{2} / M^{2} ; \alpha_{\mathrm{s}}\right)$.

Analytic expressions for $h_{N}(\gamma)$ are given in refs. $[2,3,6]$.

Eq. (9) is consistent with the results of the QCD factorization theorem of mass singularities [1]. The gluon density $G_{N}\left(\mu^{2}\right)$ is indeed factorized with respect to the coefficient function $C_{N}$. The latter is computable in QCD perturbation theory and eq. (10) provides explicitly its resummed expression (including the dependence on the factorization scale $\mu^{2}$ ) to the leading order $\left(\alpha_{\mathrm{s}} / N\right)^{k}$. The resummation effect is incorporated in eq. (10) through the $\alpha_{\mathrm{s}} / N$ dependence of $\gamma_{N}$ known from eq. (6) and the $\gamma$ dependence of $h_{N}(\gamma)$ as given by eq. (11).

We have so far reviewed the general results of the high-energy factorization, showing its consistency with the factorization theorem of mass singularities. The main point we would like to address in the rest of the paper is how exactly the two factorization theorems compare with each other. The reason for that is twofold. First, this issue is relevant to extend the valıdity of high-energy factorization to subleading orders. More importantly, the question is essentral for precise phenomenological predictions at finite energies when one has to combine the resummed coefficient function (10) with fixed-order non-logarithmic contributions computed with traditional methods [11].

Stated differently, we have to relate precisely the factorization scale $\mu^{2}$ appearing in eq. (5), or equivalently the gluon density $G\left(\mu^{2}\right)$, to the corresponding scale and density as defined in a well prescribed scheme of regularization and factorization of mass singularities.

Note however, that the question is no longer rele- 
vant if one limits oneself to considering only ratios of cross sections, like for instance the heavy-flavour photoproduction and leptoproduction cross sections. In this case, the gluon density and the factorization scale dependence cancel in the ratio, thus leading to an absolute prediction in terms of hard scales and $h_{N}(\gamma)$ functions of the type in eq. (11) [3].

\section{The gluon Green function}

In order to define the contents of eq. (5) precisely, we have to regularize the collinear (and infrared) d1vergences appearing in the perturbative QCD calculation at parton level. We proceed in a standard way using dimensional regularization, performing our calculation in $n=4+2 \epsilon$ space-tıme dimensions and considering $(n-2)$ physical polarization states of the gluon. In the following, $\mu^{2}$ will denote the dimensional regularization scale.

In the $k_{\perp}$-factorization formula (2) we have thus to introduce the replacement

$\mathbf{d}^{2} \boldsymbol{k} \mathscr{F}_{N}(\boldsymbol{k}) \rightarrow \mathrm{d}^{2+2 \epsilon} \boldsymbol{k} \mathscr{F}_{N}\left(\boldsymbol{k} ; \mu^{2}, \boldsymbol{\epsilon}\right) G_{N}^{(\mathrm{B})}\left(\mu^{2}, 1 / \boldsymbol{\epsilon}\right)$.

Here the gluon Green function $\mathscr{F}_{N}\left(\boldsymbol{k} ; \mu^{2}, \epsilon\right)$ denotes the full $g(p)+g(-k) \rightarrow g(-p)+g(k)$ absorptive part evaluated in $n=4+2 \epsilon$ dimensions and $G_{N}^{(B)}\left(\mu^{2}\right.$, $1 / \epsilon)$ is the bare gluon density.

In the limit $\epsilon \rightarrow 0$ the gluon Green function $\mathscr{F}_{N}(\boldsymbol{k}$; $\left.\mu^{2}, \epsilon\right)$ has poles $\left(\alpha_{\mathrm{s}} / \epsilon\right)^{k}$ which, according to the renormalization group, can be factorized as follows:

$$
\begin{aligned}
& \mathscr{F}_{N}\left(\boldsymbol{k} ; \mu^{2}, \boldsymbol{\epsilon}\right)=\frac{\gamma_{N}\left(\alpha_{\mathrm{s}}\right)}{\pi \boldsymbol{k}^{2}} \widetilde{R}_{N}\left(\boldsymbol{k}, \mu_{\overline{\mathrm{MS}}}, \alpha_{\mathrm{s}} ; \mu, \boldsymbol{\epsilon}\right) \\
& \quad \times \Gamma_{N}\left(\alpha_{\mathrm{s}} S_{\epsilon}\left(\mu_{\overline{\mathrm{MS}}} / \mu\right)^{2 \epsilon}, 1 / \boldsymbol{\epsilon}\right),
\end{aligned}
$$

where

$\Gamma_{N}\left(\alpha_{\mathrm{s}}, 1 / \epsilon\right)=\exp \left(\frac{1}{\epsilon} \int_{0}^{\alpha_{\mathrm{s}}} \frac{\mathrm{d} \alpha}{\alpha} \gamma_{N}(\alpha)\right) ;$

$\gamma_{N}\left(\alpha_{\mathrm{s}}\right)$ is the gluon anomalous dimension and the function $\widetilde{R}_{N}$ has no poles in $1 / \epsilon$. Performing the collinear pole factorization in eq. (13), we have introduced a factorization scale $\mu_{\mathrm{MS}}$ different from the dimensional regularization scale $\mu$. The subscript $\overline{\mathrm{MS}}$ means that we have chosen the modified minimal subtraction scheme (note the factor $S_{\epsilon}=$ $\exp \{-\epsilon[\psi(1)+\ln 4 \pi]\}$ in the argument of the factorized function $\Gamma_{N}$ in eq. (13)).

The singular (in the $\epsilon \rightarrow 0$ limit) function $\Gamma_{N}$ in eq. (14) is then used to renormalize the bare gluon density $G_{N}^{(\mathbf{B})}$, defining the $\overline{\mathrm{MS}}$-scheme gluon structure function,

$$
\begin{aligned}
& G_{N}^{(\overline{\mathrm{MS}})}\left(\mu_{\mathrm{MS}}^{2}\right) \\
& \quad=\Gamma_{N}\left(\alpha_{\mathrm{s}} S_{\epsilon}\left(\mu_{\overline{\mathrm{MS}}} / \mu\right)^{2 \epsilon}, 1 / \epsilon\right) G_{N}^{(\mathrm{B})}\left(\mu^{2}, 1 / \epsilon\right),
\end{aligned}
$$

and thus leaving a residual $\widetilde{R}_{N}$ dependence in the $\overline{\mathrm{MS}}$-scheme coefficient function.

In order to compute $\tilde{R}_{N}$ (and $\Gamma_{N}$ ) in the high-energy limit, we have to evaluate the Green function $\mathscr{F}_{N}\left(k ; \mu^{2}, \epsilon\right)$, resumming all the $\left(\alpha_{\mathrm{s}} / N\right)^{k}$ contributions. In the case of $n=4$ dimensions the resummation is accomplished by the BFKL equation [8]. The analogous equation in $n$ space-time dimensions is the following:

$$
\begin{aligned}
& \mathscr{F}_{N}\left(\boldsymbol{k} ; \mu^{2}, \boldsymbol{\epsilon}\right)=\delta^{(2+2 \epsilon)}(\boldsymbol{k})+\frac{\bar{\alpha}_{\mathrm{s}}}{N} \int \frac{\mathrm{d}^{2+2 \epsilon} \boldsymbol{q}}{(2 \pi \mu)^{2 \epsilon}} \frac{1}{\pi \boldsymbol{q}^{2}} \\
& \times\left(\mathscr{\mathscr { T }}_{N}\left(\boldsymbol{k}-\boldsymbol{q} ; \mu^{2}, \epsilon\right)-\frac{\boldsymbol{k} \cdot(\boldsymbol{k}-\boldsymbol{q})}{(\boldsymbol{k}-\boldsymbol{q})^{2}} \mathscr{\mathscr { F }}_{N}\left(\boldsymbol{k} ; \mu^{2}, \boldsymbol{\epsilon}\right)\right) .
\end{aligned}
$$

We have derived eq. (16) by generalizing the soft gluon insertion technique in refs. [12-14] to the case of $n$ dimensions. In this approach the $(k+1)$-loop contribution to the discontinuity of the gluon forward-scattering amplitude $\mathscr{F}$ is written in terms of a soft gluon factor times the $k$ th-order contribution to the amplitude itself. Using this recurrence relation and following exactly the same steps as in refs. [12,14] one obtains eq. (16).

Some comments are in order. In the case of $n=4$ dimensions $(\epsilon=0)$, after azimuthal average over $\boldsymbol{q}$, eq. (16) reproduces the BFKL equation $[8,9]$. Moreover, the integrand of the homogeneous term in (16) is exactly the same as for the BFKL equation. This is because, in the calculation of the gluon forward-scattering amplitude, a single gluon polarization dominates to leading-logarithmic order in the high-energy limit. Thus, the relevant QCD matrix elements are the same as for $n=4$ and the only difference comes from the $n$-dimensional phase-space integrations.

Note, however, an essential difference with respect 
to the BFKL equation: the kernel of eq. (16) is not scale-invariant. Indeed scale invariance is broken by dimensional regularization and the eigenfunctions of the kernel are no longer simple powers $\left(\boldsymbol{k}^{2}\right)^{\gamma}$. From a physical viewpoint this means that whilst small and large transverse-momentum regions contribute equally to the BFKL equation, they are now weighted asymmetrically. Thus, the symmetry of the gluon anomalous dimension (6) under $\gamma \rightarrow 1-\gamma$ (see eq. (7)) is expected not to be valid for the residual function $\tilde{R}_{N}$.

Before we present the solution of the gluon Green function equation (16), we should comment on other approaches to relate high-energy factorization to factorization of mass singularities. In refs. [4,5] the gluon density $\mathscr{F}(x, \boldsymbol{k})$ was obtained as the solution of the BFKL equation, respectively with a properly modified kernel and with an appropriate parametrization of the inhomogeneous term. These approaches are both legitimate as far as they amount to defining the universal density $\mathscr{F}(x, \boldsymbol{k})$ entering the high-energy factorization formula (2). However, neither solves the problem addressed in this paper, namely how $\mathscr{F}(x, \boldsymbol{k})$ compares with the gluon density as defined in standard regularization procedures of mass singularities.

As noticed above, the gluon Green function kernel in (16) is not scale-invariant and no simple technique allows us to diagonalize the integral equation. However, it is possible to solve it as a formal power series in $\alpha_{\mathrm{s}}$ with $\epsilon$-dependent coefficients, and also to obtain an explicit solution in the relevant limit of small $\epsilon$ values. The latter is sufficient to compute the singular contribution $\Gamma_{N}$ in eq. (13) and the associated function $\tilde{R}_{N}$ for $\epsilon \rightarrow 0$. More precisely, in addition to deriving again the BFKL result (6) for the gluon anomalous dimension in eq. (14), we obtain (details of the calculation will be reported elsewhere):

$$
\begin{aligned}
& \tilde{R}_{N}\left(\boldsymbol{k}, \mu_{\overline{\mathrm{MS}}}, \alpha_{\mathrm{s}} ; \mu, \epsilon=0\right)=R_{N}\left(\alpha_{\mathrm{s}}\right)\left(\frac{\boldsymbol{k}^{2}}{\mu_{\overline{\mathrm{MS}}}^{2}}\right)^{\gamma_{N}\left(\alpha_{\mathrm{s}}\right)}, \\
& R_{N}\left(\alpha_{\mathrm{s}}\right)=\left(\frac{\Gamma\left(1-\gamma_{N}\right) f\left(\gamma_{N}\right)}{\Gamma\left(1+\gamma_{N}\right)\left[-\gamma_{N} f^{\prime}\left(\gamma_{N}\right)\right]}\right)^{1 / 2} \\
& \quad \times \exp \left(\gamma_{N} \psi(1)+\int_{0}^{\gamma N} \mathrm{~d} \gamma \frac{\psi^{\prime}(1)-\psi^{\prime}(1-\gamma)}{f(\gamma)}\right),
\end{aligned}
$$

where $f$ and $f^{\prime}$ are the characteristic function in eq.
(7) and its first derivative, respectively. The $\alpha_{\mathrm{s}}$-dependence of the RHS in (18) is implicit in that of the BFKL anomalous dimension $\gamma_{N}=\gamma_{N}\left(\alpha_{\mathrm{s}}\right)$. Note that for $\epsilon \rightarrow 0$ any residual dependence on the dimensional regularization scale $\mu$ consistently disappears from the result (17).

\section{High-energy factorization and mass-singularity factorization}

We are now in a position to relate the high-energy factorization formula (2) to the factorization theorem of mass singularities in the $\overline{\mathrm{MS}}$ scheme [1]. Performing the replacement (12) in eq. (2) and factorizing mass singularities according to eqs. (13) and (14), we obtain

$$
\begin{aligned}
& 4 M^{2} \sigma\left(\rho, M^{2}\right) \\
& =\int_{0}^{1} \frac{\mathrm{d} z}{z} C^{(\overline{\mathrm{MS}})}\left(\rho / z, \alpha_{\mathrm{s}}, M^{2} / \mu_{\overline{\mathrm{MS}}}^{2}\right) G^{(\overline{\mathrm{MS}})}\left(z, \mu_{\overline{\mathrm{MS}}}^{2}\right),
\end{aligned}
$$

or, equivalently, in $N$-moment space

$$
\begin{aligned}
& 4 M^{2} \sigma_{N}\left(M^{2}\right) \\
& \quad=C_{N}^{(\overline{\mathrm{MS}})}\left(\alpha_{\mathrm{s}}, M^{2} / \mu_{\overline{\mathrm{MS}}}^{2}\right) G_{\bar{N}}^{(\overline{\mathrm{MS}})}\left(\mu_{\mathrm{MS}}^{2}\right),
\end{aligned}
$$

where the (resummed) $\overline{\mathrm{MS}}$-scheme coefficient function $C_{N}^{(\overline{\mathrm{MS}})}$ is given in terms of the functions $h_{N}$ and $R_{N}$ in eqs. (11) and (18),

$$
\begin{aligned}
& C_{N}^{(\overline{\mathrm{MS}})}\left(\alpha_{\mathrm{s}}, M^{2} / \mu_{\overline{\mathrm{MS}}}^{2}\right) \\
& =h_{N}\left(\gamma_{N}\left(\alpha_{\mathrm{s}}\right)\right) R_{N}\left(\alpha_{\mathrm{s}}\right)\left(\frac{M^{2}}{\mu_{\mathrm{MS}}^{2}}\right)^{\gamma_{N}\left(\alpha_{\mathrm{s}}\right)}
\end{aligned}
$$

This result can be stated in a different but equivalent way by saying that the unintegrated gluon density $\mathscr{F}^{\circ}(x, \boldsymbol{k})$ in the $k_{\perp}$-factorization formula (2) is precisely related to the customary gluon structure function $G_{N}^{(\overline{\mathrm{MS}})}$ in the $\overline{\mathrm{MS}}$-scheme through the following relation (in $N$-moment space):

$$
\mathscr{F}_{N}(\boldsymbol{k})=\frac{\gamma_{N}\left(\alpha_{\mathrm{s}}\right)}{\pi \boldsymbol{k}^{2}} R_{N}\left(\alpha_{\mathrm{s}}\right)\left(\frac{\boldsymbol{k}^{2}}{\mu_{\mathrm{MS}}^{2}}\right)^{\gamma_{N}\left(\alpha_{\mathrm{s}}\right)} G_{N}^{(\overline{\mathrm{MS}})}\left(\mu_{\overline{\mathrm{MS}}}^{2}\right) .
$$

The $\overline{\mathrm{MS}}$-scheme coefficient function in eq. (21) differs from $C_{N}$ in eq. (10) by the process-independent factor $R_{N}$. As discussed in section $1, R_{N}$ can in 
principle be re-absorbed in the definition of the gluon density by changing the factorization scheme, i.e. considering the gluon structure function $G_{N}\left(\mu^{2}\right)$ as defined by eq. (3). Note however that the anomalous dimension has to be modified accordingly. Denoting by $\tilde{\gamma}_{N}\left(\alpha_{\mathrm{s}}\right)$ the new anomalous dimension

$\frac{\mathrm{d} \ln G_{N}\left(\mu^{2}\right)}{\mathrm{d} \ln \mu^{2}}=\tilde{\gamma}_{N}\left(\alpha_{\mathrm{s}}\right)$,

we have

$\tilde{\gamma}_{N}\left(\alpha_{\mathrm{s}}\right)=\gamma_{N}^{(\overline{\mathrm{MS}})}\left(\alpha_{\mathrm{s}}\right)+\beta\left(\alpha_{\mathrm{s}}\right) \frac{\mathrm{d} \ln R_{N}\left(\alpha_{\mathrm{s}}\right)}{\mathrm{d} \ln \alpha_{\mathrm{s}}}$,

where $\beta\left(\alpha_{\mathrm{s}}\right)=\mathrm{d} \ln \alpha_{\mathrm{s}} / \mathrm{d} \ln \mu^{2}$ and $\gamma_{N}^{(\overline{\mathrm{MS}})}\left(\alpha_{\mathrm{s}}\right)$ is the complete gluon anomalous dimension in the $\overline{\mathrm{MS}}$ scheme and not only its leading-order expression as given by the BFKL equation (6).

The function $R_{N}$, although resumming high-energy leading contributions $\left(\alpha_{s} / N\right)^{k}$ to all orders, differs from unity only in the third perturbative order:

$$
\begin{aligned}
& R_{N}\left(\alpha_{\mathrm{s}}\right)=1+\frac{8}{3} \zeta(3)\left(\frac{\bar{\alpha}_{\mathrm{s}}}{N}\right)^{3}-\frac{3}{4} \zeta(4)\left(\frac{\bar{\alpha}_{\mathrm{s}}}{N}\right)^{4} \\
& +\frac{22}{5} \zeta(5)\left(\frac{\bar{\alpha}_{\mathrm{s}}}{N}\right)^{5}+\mathrm{O}\left(\left(\frac{\bar{\alpha}_{\mathrm{s}}}{N}\right)^{6}\right) .
\end{aligned}
$$

Therefore its effect on the $\overline{\mathrm{MS}}$-scheme coefficient function (21) is expected not to be numerically relevant up to very small values of $x$, say $x \leqq 10^{-3}$.

Note however that $R_{N}$ is singular at the saturation value $\gamma_{N}=\frac{1}{2}$ of the BFKL anomalous dimension $[8,3]$ :

$R_{N}\left(\alpha_{\mathrm{s}}\right) \simeq$ const. $\left(\frac{1}{1-2 \gamma_{N}\left(\alpha_{\mathrm{s}}\right)}\right)^{1 / 2}$

$$
\left(\gamma_{N} \rightarrow \frac{1}{2}\right) \text {. }
$$

This behaviour is related to the branch-point singularity of the BFKL anomalous dimension at $N=$ $4 \bar{\alpha}_{\mathrm{s}} \ln 2$ and signals the ultimate failure of the perturbative approach to the mass singularity factorization and the onset of the multi-Regge factorization at extreme energies (see ref. [3] for a more detailed discussion of this issue).

As pointed out in refs. $[4,6]$, precise phenomenological predictions for hard-scattering cross sections at collider (finite) energies require careful matching of the resummed leading $\ln x$-contributions (accomplished by the high-energy factorization) with non- logarithmic terms. The latter are computed in fixedorder perturbation theory, using the factorization theorem of mass singularities. The relation between high-energy and mass-singularity factorizations established in this paper allows one to combine resummed and fixed-order expressions avoiding any mis-counting. In the case of heavy-flavour photoproduction, a detailed matching procedure was performed in refs. [6,7], however without taking into account the $\overline{\mathrm{MS}}$-scheme correction factor $R_{N}$. We checked numerically that $R_{N}$ has no sizeable effects in that case, thus leaving unchanged our conclusions in ref. [6] about heavy-flavour production at HERA energies. Nevertheless, the effect of $R_{N}$ for higher energies and other processes (in particular heavy-flavour hadroproduction) has to be investigated.

We conclude the paper with a comment on the case of a running coupling constant. Strictly speaking, the runnıng coupling effects mix with subleading $\ln x$ contributions. However, we can introduce the running coupling in our resummed formula (21) for the $\overline{\mathrm{MS}}$-scheme hard coefficient function by simply implementing renormalization group arguments. In the simpler case where the renormalization and the factorization scales $\mu_{\mathrm{R}}$ and $\mu_{\mathrm{F}}$ are identıfied $\left(\mu_{\mathrm{R}}=\mu_{\mathrm{F}}=\right.$ $\left.\mu_{\overline{\mathrm{MS}}}\right)$, we have

$$
\begin{aligned}
& C_{N}^{(\overline{\mathrm{MS}})}\left(\alpha_{\mathrm{s}}\left(\mu_{\mathrm{MS}}^{2}\right), M^{2} / \mu_{\mathrm{MS}}^{2}\right)=h_{N}\left(\gamma_{N}\left(\alpha_{\mathrm{s}}\left(M^{2}\right)\right)\right) \\
& \quad \times R_{N}\left(\alpha_{\mathrm{s}}\left(M^{2}\right)\right) \exp \left(\int_{\mu_{\overline{\mathrm{MS}}}^{2}}^{M^{2}} \frac{\mathrm{d} q^{2}}{q^{2}} \gamma_{N}\left(\alpha_{\mathrm{s}}\left(q^{2}\right)\right)\right) .
\end{aligned}
$$

Eq. (27) can be used for easier comparison with standard formulae obtained by using mass-singularity factorization in fixed-order perturbation theory.

\section{Acknowledgement}

We are grateful to B.R. Webber for valuable comments.

\section{References}

[1] J.C. Collıns, D E. Soper and G Sterman, in: Perturbative quantum chrodynamics, ed. A H. Mueller (World Scientıfic, Singapore, 1989), and references therein. 
[2] S. Catani, M. Ciafaloni and F. Hautmann, Phys. Lett. B 242 (1990) 97.

[3] S. Catanı, M. Ciafalonı and F Hautmann, Nucl. Phys. B 366 (1991) 135.

[4] J.C Collıns and R.K. Ellis, Nucl. Phys. B 360 (1991) 3.

[5] E.M. Levin, M.G. Ryskın, Yu.M. Shable'skii and A G Shuvaev, Sov. J. Nucl. Phys. 53 (1991) 657.

[6] S. Catanı, M. Ciafalonı and F. Hautmann, in: Proc. HERA Workshop, eds. W. Buchmuller and G. Ingelman (DESY, Hamburg, 1991) p. 690.

[7] S. Catanı, M Crafalonı and F. Hautmann, Nucl. Phys B (Proc Supp.) 29A (1992) 182

[8] L.N. Lipatov, Sov J. Nucl. Phys. 23 (1976) 338;

E.A. Kuraev, L N. Lipatov and V.S. Fadin, Sov. Phys. JETP 45 (1977) 199,

Ya. Balıtskı1 and L.N. Lıpatov, Sov. J. Nucl. Phys. 28 (1978) 822.
[9] A. Bassetto, M. Clafalonı and G. Marchesinı, Phys. Rep. 100 (1983) 201.

[10] L.V. Gribov, E.M. Levin and M.G. Ryskin, Phys. Rep. 100 (1983) 1.

[11] P Nason, S. Dawson and R.K. Ellis, Nucl. Phys. B 303 (1988) 607;

R.K. Ellis and P. Nason. Nucl. Phys. B 312 (1989) 551;

W. Beenakker, W.L. van Neerven, R. Meng, G.A. Schuler and J. Smith, Nucl. Phys. B 351 (1991) 507;

E. Laenen, S. Ruemersma, J. Smuth and W.L. van Neerven, Stony Brook preprint ITP SB 92-09.

[12] M. Clafaloni, Nucl Phys. B 296 (1987) 249.

[13] S. Catan1, F. Fioranu and G. Marchesını, Nucl. Phys. B 336 (1990) 18.

[14] S Catani, F. Fioran1, G. Marchesinı and G. Orıani, Nucl. Phys. B 361 (1991) 645. 